\title{
A Study on Fruit Setting Model of Parent Branch in Nectarine Tree
}

\author{
Ning Xia* and Aishuang Li
}

\author{
Institute of Agricultural economy, Guangdong Agricultural Academy of Sciences, 510640 China
}

\begin{abstract}
The fruit setting models of individual parent branch in 10-year-old nectarine tree (Prunus persica var. carminum) with different heading intensities were studied over two successive growth seasons (2016-2017). Parent branches after being headed, which is comprised of a succession of nodes with zero to two flower buds which can produce fruits, is characterized by a Markov chain with three states, and therefore the corresponding model parameters consists of initial probability together with the following transition probabilities of states (fruit setting types): state 2 represents two fruits on bearer node; state 1 represents one fruit on bearer node; state 0 represents no fruit on bearer node. On each node, the numbers of fruits and their spatial arrangement per parent branches follow a definite pattern that remains the same as long as the selective heading intensity is kept constant, i.e. that fruit bearing was significantly higher in the mesotonic zone of the shoot and lower in the basal and apical zone. The simulation results with Markov chain indicate that this final fruit set sequence was correlated with the heading activity. Three-dimensional representations of the fruit setting processes using Pruningsim software illustrate more vividly perhaps than the numbers of fruit occurrence being listed in a table and/or figure. The visual comparison between 3D reconstructions and realistic photographs may generate a natural feedback of the pruning measurements, at the shoot scale.
\end{abstract}

Keywords: Markov chain, Visualization, Heading, Architectural analysis.

\section{INTRODUCTION}

During the last three decades, the advent of increasingly powerful PCs enabled plant models to be developed [24] using algorithms, such as computer grammars or stochastic processes [5]. With significant advancements in tree models [15], it not only can study the structure and the nature of each organ at any given time of the tree's life to reveal some underlying physiological mechanisms of its growth and development [16], but also optimize empirical management practices for improving fruit productivity and quality. Thus, modeling, simulation and visualization of the plant have become major research tools in understanding plant growth, flowering and fruiting processes. These technologies are extremely important for tree research because they can reconstruct the structure and function of a tree using rules extracted from real tree in short period of time as compared to its long lifespans from decade to century [32].

Several types of architectural tree models have been developed during the last four decades. Of these, excellent progresses have been made in dynamic modeling the branching patterns of tree $[6,8,10,25$, 31] using Markov chains. The distributions of lateral shoot along parent branch have been simulated in several fruit tree species, such as apple, plum, peach, and kiwifruit.

"Address correspondence to this author at the Institute of Agricultural economy, Guangdong Agricultural Academy of Sciences, 510640 China;

Tel: 0862038319940; Fax: 0862038319940; E-mail: nxia01@sina.com
The evidence that numerous flowers produced and smaller number of fruits formed are common in fruit tree species [1]. Often, percentages of fruit set are controlled by different genetic, psychological, environmental and cultural factors [28] such as scion cultivars, rootstocks, air temperature, training and pruning [11], however, not all of them provide same influence on each node with flower buds, thus the fruits produced are not distributed uniformly within the tree canopy.

It is well known that fruit spatial distribution within tree canopy has distinct impact on shape, weight, firmness, texture, taste, and flavor [27].

There have been many studies on how to quantitatively analyze patterns of variation in fruit characteristics over recent decades $[3,4,26]$. Smith and colleagues had measured accurately the spatial coordinates of each fruit on a kiwifruit vine and reconstructed 3-D mode in which fruit quality attributes can be distinguished formally [27]. Godin et al. (1998) also developed novel encoding model to investigate 3D structural properties of fruit [10]. Hall and Gandar (1996) use stochastic model to simulate the dynamic of fruit growth [14]. However, the topological information of each fruit in tree canopy, which is essential for the study of patterns of fruit set, is not available for abovementioned methods.

The number of mature fruits per nodes on parent branches is also a key indicator of the bearing habit of trees, which can to some extent determine both fruit quality and fruit yield [22]. 
The occurrence and spatial distribution of bearing shoots of fruit trees in traditional orchard can be adjusted by various techniques such as thinning, heading back and topping at different times.

The relative efficiencies of the technological and economic benefits of them on some fruit tree varieties had been evaluated through comparative analysis $[9,18,23,30]$. However, it is difficult to make factor comparisons about the number of fruits on parent branches under different pruning measures. There are physiological factors as structure defect of flowers, inadequate nutrition, deficient pollination, and also environmental factors such as high or low temperature, weak light, low soil moisture, and improper pruning practices. Although some studies have investigated relationships between fruit set and position of flower on parent branch [21], unfortunately, there is little study on the fruit setting patterns of individual parent branch with different pruning intensities [20].

Effects of different heading intensities on fruit set of parent branch were modeled with Markov chains models. The method provides optimum decision making for heading before you can visually determine the fruit number in each of the nodes and the sequence of the fruits along each parent branch [17].

Furthermore, 3-D structure of each parent branch can be built using Pruningsim [33], in which virtual parent branch may be viewed and manipulated from any angle.

\section{MATERIALS AND METHODS}

\subsection{Experimental Site}

The experiment in complete randomized block design was carried out in December 2017 on a commercial orchard in Jiufeng, Guangdong, China (113 deg $35^{\prime}$ E, 25 deg 14' N, $800 \mathrm{~m}$ above sea level). The mean annual air temperature in this area is about 18.6 ${ }^{\circ} \mathrm{C}$ and the soil is classified as brown earth. Each plot was comprised of 16 trees planted in a four-row $x$ fourtree array with north-south orientation, so that the middle three trees of the middle row were completely guarded.

\subsection{Tree Description}

Nectarine (Prunus persica var. carminum) tree were planted at a spacing of $3 \times 2 \mathrm{~m}$ with a density of 1665 trees/ha. The current-year shoots that had formed in the 2016 growing season were called parent branches.
All samples of 1026 parent branches were randomly selected from 50 trees with different heading intensities.

Heading refers to the removal of $s$ portion of a parent branch to a node with buds. Three heading intensities, light, medium and severe heading were available in horticulture.

In this paper, 3 nodes retained per parent branch after heading was called $3 \mathrm{nh}$ for short, 4 nodes retained per parent branch after heading was called $4 \mathrm{nh}$, and so on. All treatments were further divided into three heading intensities, light, medium and severe heading. Of these, ' $4 \mathrm{nlh}$ ' and ' $5 \mathrm{nlh}$ ' were of severe heading, " $6 \mathrm{nlh}$ " to " $14 \mathrm{nlh}$ " were of the same heading intensity as medium heading back, and " $15 \mathrm{nlh}$ " and more were classified as light heading.

To determine whether pruning practices affect the fruiting pattern within the pruned parent branch, censuses of fruit sampling were made at the harvest period of 2016-2017. The number of fruits were counted according to the position of the node on each parent branch.

\subsection{Model Description}

Each node on a parent branch after heading may have from zero to 3 buds. Three buds, two flower buds and a leaf bud, are visual at each node of strong parent branch after heading, except for the basal node. In contrast, weak parent branch may have only 0 to 2 buds on each node. A flower bud can set zero to one fruit. Therefore, each node has zero, one or two fruits that constitutes natural sequential structure from which Markov chain can be built.

Markov chain is a computational method to model consecutive sequence of states where it is assumed that the current states depend entirely on the previous state according to given probabilistic rules.

The states of model are defined as follows: state 0 that is zero fruit set on a node, state 1 that is one fruit set on a node, state 2 that is two fruit set on a node. Three-state Markov model has two parameters, initial probability $(\pi)$ and transition probability $(p)$ of the state.

The initial probability of state $1\left(\pi_{1}\right)$ denotes that one fruit sets at the basal node. Like this, $\pi_{2}$ denotes that two fruits set at the basal node, and $\pi_{0}$ denotes that zero fruit sets at the basal node. The sum of the initial probabilities of three states must be equal to one. 
Transition probability of the state $(p)$ is probability of going from the current state to next state, and for which the probability of the next state depends only on the current one, not on the previous one (Figure 1).

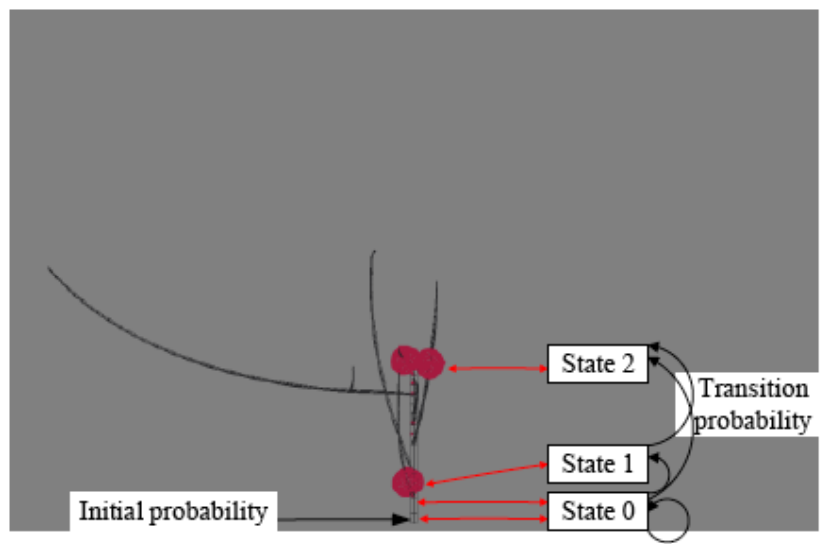

Figure 1: State diagram of Markov chain. This is the example of "10nlh". The transition probability the state is represented by the broken line and the initial probability of the state by the continuous line.

Maximum Likelihood method is used to estimate the parameter values which are most likely to generate the observed data [31].

The topological property of fruit setting can be characterized by a series of frequency distribution of the state as a function of the number of nodes [12].

The characteristics of interval distribution consists of the two following types: recurrence distribution, which is defined as the number of nodes where a given state has occurred before it occurs again; and sojourn distribution, which is defined as the number of successive nodes where a given state occurs again.

\section{RESULTS}

\section{Effects of Heading Intensities on the Percentage and Total Number of Fruits on Parent Branches}

The results showed that $5 \%$ nodes on ' $8 \mathrm{nlh}$ ' parent branch were in the state 2 which were significantly $(P=$ 0.04) greater than those on ' $15 \mathrm{nlh}$ ' parent branch, which on average was $0.8 \%$ (from $11^{\text {th }}$ to $15^{\text {th }}$ node), $1.5 \%$ (from $6^{\text {th }}$ to $10^{\text {th }}$ node), and $0.3 \%$ (from $1^{\text {st }}$ to $5^{\text {th }}$ node) respectively.

We also computed the occupancy distribution of state 1 on ' $15 \mathrm{nlh}$ ' parent branch, with on average 0.72 , 1.2 , and 0.48 of nodes occurrence at the apical, intermediate, basal portion of parent branches, respectively.
Basal nodes were kept in state 0 by positional cues that were largely insensitive to light heading. Intermediate nodes were increasingly able to set fruits, with the most fruits observed from $6^{\text {th }}$ to $10^{\text {th }}$ node in our results, and less fruit set was observed at nodes closer to the apex, with 15 to $20 \%$ of these nodes set fruits and 77 to $84 \%$ of nodes retained in the state 0 .

The probability of fruit set for parent branches with ' $15 \mathrm{nlh}$ ' was close to 0.34 , the mean number of fruits harvested was 5.0.

Compared with light heading and intermediate heading, the occupancy distributions of the state 1 and state 0 on parent branches with ' $6 \mathrm{nlh}$ ' to ' $9 \mathrm{n} l \mathrm{l}$ ' heading were uniform while that of the state 2 were heterogeneous suggesting that the probability of state 2 was the highest near the median node $(2.1 \%)$, at apical nodes it was $0.6 \%$ (from $7^{\text {th }}$ to $8^{\text {th }}$ node), and at a proximal nodes (from $1^{\text {st }}$ to $3^{\text {rd }}$ node) it was 0 , the probability of the state 1 varied from $51.3 \%$ at the median nodes, $24.7 \%$ at apical nodes, to $11.7 \%$ at the proximal nodes. The probability of fruit set was on average greater on parent branches with medium heading than those with severe heading, which was attributed to potential benefit that was more conducive for reproductive growth.

Table 1: Estimates of Mean State Occupancy Distribution and Mean ( \pm s.d.) Number of Fruits along Parent Branches with Different Heading Intensities

\begin{tabular}{|cccccc|}
\hline $\begin{array}{c}\text { Heading } \\
\text { intensities }\end{array}$ & \multicolumn{2}{c}{$\begin{array}{c}\text { Mean state occupancy } \\
\text { distribution }\end{array}$} & $\begin{array}{c}\text { Mean fruit } \\
\text { number }\end{array}$ & $\begin{array}{c}\text { Probability } \\
\text { of fruit set }\end{array}$ \\
\hline \hline \multirow{2}{*}{$4 \mathrm{nlh}$} & State 0 & State 1 & State 2 & & \\
$5 \mathrm{nlh}$ & $2.8 \pm 0.2$ & $1.0 \pm 0.1$ & $0.0 \pm 0.0$ & 0.8 & 0.20 \\
$6 \mathrm{nlh}$ & $4.7 \pm 0.3$ & $1.3 \pm 0.2$ & $0.0 \pm 0.0$ & 1.1 & 0.21 \\
$7 \mathrm{nlh}$ & $4.9 \pm 0.2$ & $1.9 \pm 0.4$ & $0.2 \pm 0.0$ & 1.9 & 0.27 \\
$8 \mathrm{nlh}$ & $5.4 \pm 0.4$ & $2.1 \pm 0.3$ & $0.4 \pm 0.1$ & 2.1 & 0.26 \\
$9 \mathrm{nlh}$ & $6.2 \pm 0.6$ & $2.4 \pm 0.2$ & $0.3 \pm 0.0$ & 2.6 & 0.29 \\
$15 \mathrm{nlh}$ & $11.0 \pm 0$. & $3.6 \pm 0.3$ & $0.3 \pm 0.1$ & 5.0 & 0.34 \\
\hline
\end{tabular}

The difference in fruit number of parent branches with severe heading was much smaller that ranged from 0.8 for ' $4 \mathrm{nlh}$ ' to 1.1 for ' $5 \mathrm{nlh}$ ', and similar results also were showed on parent branch with medium heading that ranged from 1.5 for ' $6 \mathrm{nlh}$ ' to 2.6 for ' $9 \mathrm{nlh}$ '. The mean number of the state 0 on parent branch with ' $15 \mathrm{nlh}$ ' is $11.0 \pm 0.5$ whereas that of the state 1 and $2 \mathrm{did}$ 
not increase significantly. Finally, $24 \%$ of the nodes were in the state 1 which corresponded to 3.6 fruits on parent branches with light heading, $28 \%$ of the nodes were in the state 1 which corresponded to 2 fruits on parent branches with medium heading, and $25 \%$ of the nodes were in the state 1 which corresponded to only one fruit on parent branches with severe heading (Table 1).

\section{Topological Characteristics of Fruit Set on Parent Branches}

Table 2: Initial and Transition Probabilities of each State on Parent Branches with Different Heading Intensities

\begin{tabular}{|c|c|c|c|c|}
\hline \multirow{2}{*}{$\begin{array}{l}\text { Heading } \\
\text { intensities }\end{array}$} & \multirow{2}{*}{$\begin{array}{c}\text { Initial } \\
\text { probabilities }\end{array}$} & \multicolumn{3}{|c|}{ Transition probabilities } \\
\hline & & State 0 & State 1 & State 2 \\
\hline \multirow{3}{*}{$4 \mathrm{nlh}$} & State 0: 1 & 0.56 & 0.44 & 0 \\
\hline & State 1: 0 & 0.87 & 0.13 & 0 \\
\hline & State 2: 0 & 0.94 & 0.06 & 0 \\
\hline \multirow{3}{*}{$5 \mathrm{nlh}$} & State 0: 1 & 0.57 & 0.43 & 0 \\
\hline & State 1: 0 & 0.89 & 0.11 & 0 \\
\hline & State 2: 0 & 0.90 & 0.10 & 0 \\
\hline \multirow{3}{*}{6 nlh } & State $0: 1$ & 0.78 & 0.21 & 0.01 \\
\hline & State 1: 0 & 0.80 & 0.20 & 0 \\
\hline & State 2: 0 & 0.78 & 0.22 & 0 \\
\hline \multirow{3}{*}{$7 \mathrm{nlh}$} & State 0: 1 & 0.76 & 0.23 & 0.01 \\
\hline & State 1: 0 & 0.77 & 0.22 & 0.01 \\
\hline & State 2: 0 & 0.75 & 0.23 & 0.02 \\
\hline \multirow{3}{*}{ 8nlh } & State 0: 1 & 0.64 & 0.34 & 0.02 \\
\hline & State 1: 0 & 0.74 & 0.25 & 0.01 \\
\hline & State 2: 0 & 0.75 & 0.22 & 0.03 \\
\hline \multirow{3}{*}{ 9nlh } & State 0: 1 & 0.74 & 0.26 & 0 \\
\hline & State 1: 0 & 0.74 & 0.24 & 0.02 \\
\hline & State 2: 0 & 0.77 & 0.23 & 0 \\
\hline \multirow{3}{*}{$15 \mathrm{nlh}$} & State $0: 1$ & 0.78 & 0.19 & 0.03 \\
\hline & State 1: 0 & 0.85 & 0.15 & 0 \\
\hline & State 2: 0 & 0.89 & 0.11 & 0 \\
\hline
\end{tabular}

Table 2 showed that the initial probabilities of the different states remained constant, which indicated that probabilities of fruit set at basal nodes of parent branches were not easily influenced by the heading intensities. Meanwhile, the transition probabilities for parent branches with severe heading remained unchanged. Apical nodes at parent branches with light and severe heading could not set fruits, as indicated by low transition probabilities from state 0 to other states. For example, the transition probabilities for ' $4 \mathrm{nlh}$ ' and ' $5 \mathrm{nlh}$ ' from state 0 to state 0 and state 0 to state 1 are both 0.6 and 0.4 , respectively, and the transition probabilities from state 0 to state 2 are 0 . Similar to the case of these transition probabilities, the data in the Table 2 showed that ' $6 \mathrm{nlh}$ ', ' $7 \mathrm{nlh}$ ', ' $8 \mathrm{nlh}$ ', and ' $9 \mathrm{nlh}$ ' had approximate transition probabilities from state 0 to other states and differed only on the transition probabilities from state 1 to state 2 and state 2 to state 2.

These results indicated that nodes at the median part of parent branches had high probability in the state 1 and state 2, but had also been found in the state 0 .

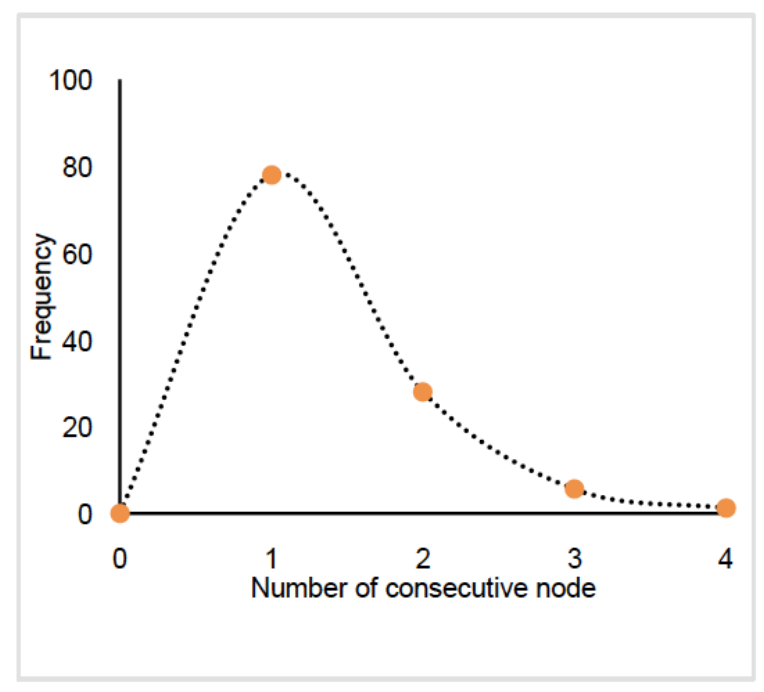

Figure 2: Sojourn distribution of state 1.

Topological analysis is one of means to identify states on parent branches through time. There are two characteristic distributions which topological analysis enjoys, both of which follow a geometric distribution in fruit settings pattern. Figure 2 below is the sojourn distribution of state 1 , which expresses the number of successive occurrences of state 1 at the nodes of ' $9 \mathrm{nlh}$ ' parent branches. Figure 3 illustrates the number of nodes starting from state 1 until its subsequent occurrence, which also is called as recurrence distribution of state 1 . Such characteristic distributions can be used to quantify the relative change in spatial position of different states on parent branches, which influence the fruit setting patterns.

Our results highlighted the isolation of fruit on ' $9 \mathrm{nlh}$ ' parent branches where the most frequent number of successive nodes were one, while the most frequent number of interval node were two. 


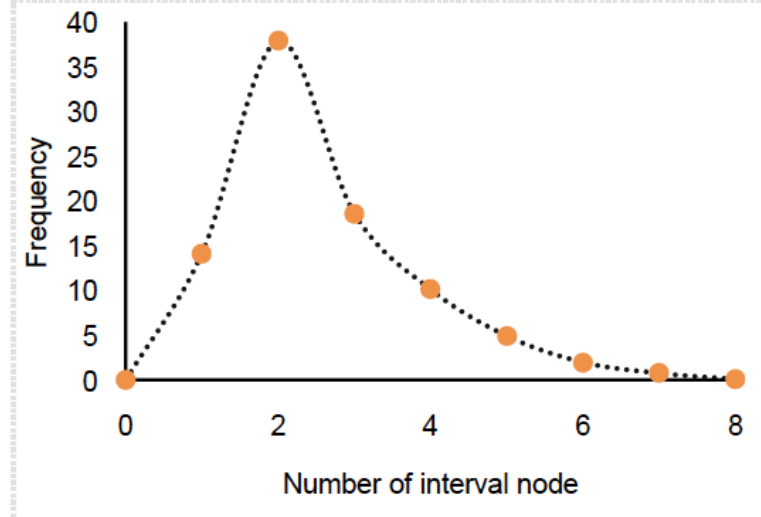

Figure 3: Recurence distribution of state 1.

\section{Interactive Simulation and 3D Visualization}

Pruningsim is a Markov model driven software tool for the simulation, visualization, and statistical analysis of fruit tree pruning, in which users can observe what the simulation output looks like.

Our results showed that distribution of fruits followed characteristic pattern. In all cases, flower buds at the mesotonic had a higher probability of fruit set than those closer to the base or the apical. For instance, parent branches with moderate heading (from "6nlh" to "9nlh") were more likely to set fruit from $3^{\text {rd }}$ to $6^{\text {th }}$ node (Figure 4).

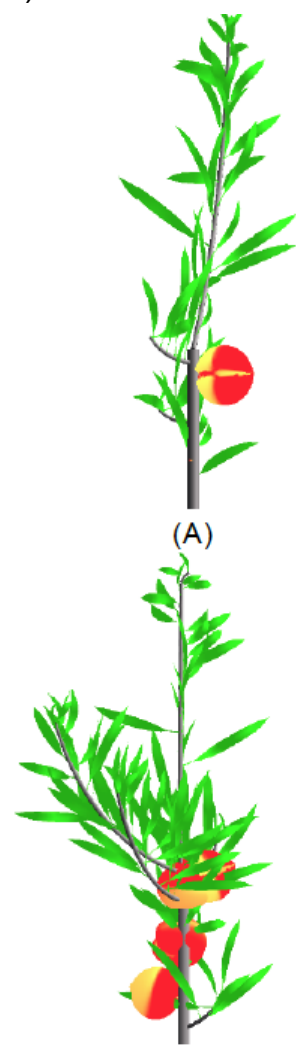

(B)

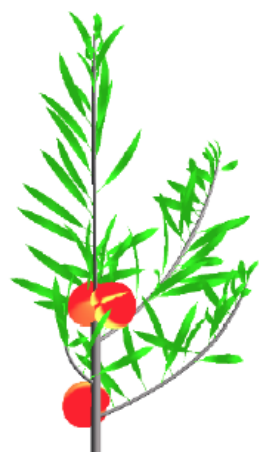

(C)

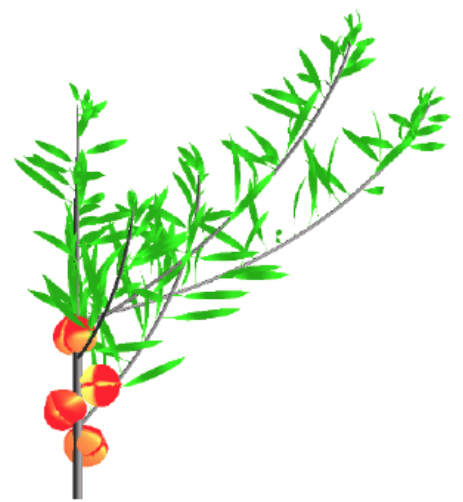

(D)

Figure 4: Simulation output for parent branches with medium heading, (A) "6nlh", (B) "7nlh", (C) "8nlh", and (D) "9nlh".

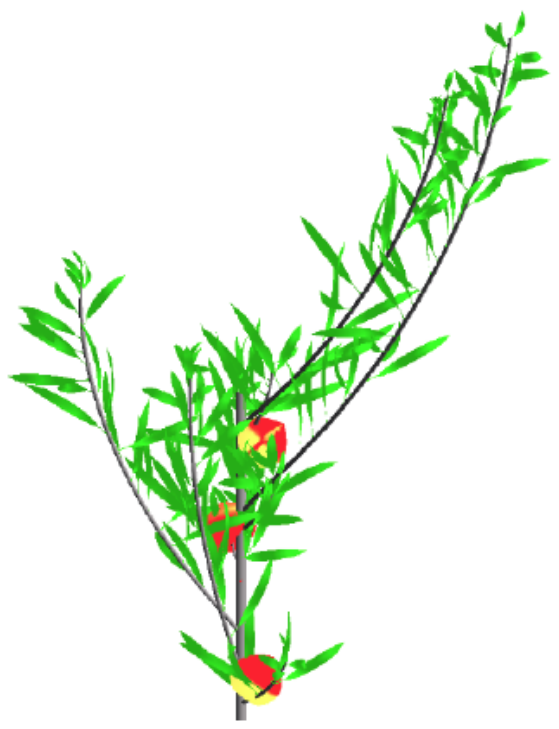

(E)

Figure 5: Simulation output for parent branches with light heading, (E) "15nlh".

Similarly, the high fruit set found from $6^{\text {th }}$ to $8^{\text {th }}$ node on parent branches with light heading (Figure 5), whereas main fruit setting position of parent branches with severe heading was $2^{\text {nd }}$ node from the proximal (Figure 6). 


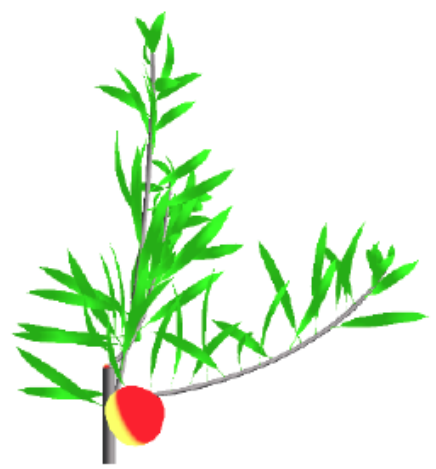

$(\mathrm{F})$

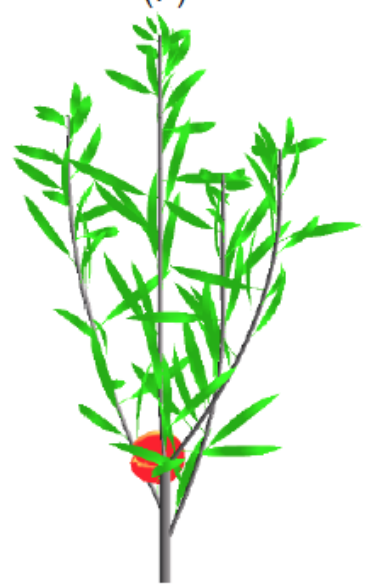

(G)

Figure 6: Simulation output for parent branches with severe heading, (F) "4nlh" and (G) "5nlh".

\section{DISCUSSIONS AND CONCLUSIONS}

The optimization of fruit set via properly pruning is well known to decrease labor costs and improve the fruit quality in intensive orchard. The fruit setting patterns may vary with complex physiological functions and morphological traits at different scales and dates, but also stochastic response of environmental factors in cultivated conditions. Little information on the architectural effect on fruit set of nectarine has been published. Thus, modeling fruit setting patterns with an emphasis on number and position of fruit along parent branches is a key step for determination of pruning strategies.

Several previous studies have introduced some hypotheses about fruit setting patterns at tree scale. Guitián proposed a "resource competition hypothesis" [13] that the fruit set might be related to limited resources for the development of fruits. This hypothesis presented a mechanistic explanation of competitive interactions between the vegetative growth and reproductive growth due to resource limit.
Brunet and Charlesworth explained the different fruit setting patterns might result from variation in pollen donors and the density of flowering individuals. They called this the "non-uniform pollination hypothesis". Although the explanation they provided was not perfect, it also made predictions of fruit set that were reasonably consistent with a number of observations made within the canopies of fruit Trees [2].

Another hypothesis assumes that various fruit setting patterns are attributable to architectural limitations on the reproductive growth aalong parent branches, which also is called as "architectural effects hypothesis" [7].

Medium heading indeed is the most widely used pruning practices in nectarine orchard. The general purpose of medium heading is not to reduce the number of node that has one or two flower buds, but to balance between vegetative and reproductive growth for stability of fruit set and high quality fruit production in successive years. Each parent branch with medium heading has two fruits on average. However, the average number of fruit on parent branch with heavy heading is less than one fruit.

Topological attributes for fruit set distribution along parent branches

The distribution of the ripe fruits on the nodes along parent branches with different heading intensities follows a fixed pattern which shows the distinguishing mesotonic traits. As we saw in the case of the simulation examples, the flower buds nearest the middle of parent branches have higher probability of fruit set than those closer to the base or the apical. This mesotonic pattern is significantly different from typical fruit setting pattern where the flower buds nearest the base of parent branches without pruning show higher fruit set.

The fruits of parent branches with ' $5 \mathrm{nlh}$ ' severe heading primarily set at $2^{\text {nd }}$ node from the proximal. Most of fruits on parent branches with '15nlh' light heading located at $6^{\text {th }}$ to $8^{\text {th }}$ node. The position of fruit set of parent branches with medium heading were from $3^{\text {rd }}$ to $6^{\text {th }}$ node. These results suggest that heading practices can affect the fruit setting patterns by controlling flower buds and regulating fruit position.

The combination of the recurrence and sojourn distributions of the states 0,1 and 2 characterized a rather scattered and diffuse fruit setting pattern. This pattern is more pronounced on light heading parent branches (see Figure 2 and Figure 3). 
Heading back also serves as a control measure for regulating fruit load. Some studies revealed that heading back reduced excessive fruit load. However, observations on nectarine showed that parent branches with regular heading back usually motivated new shoots which were out of balance in branching and fruiting especially when large, mature trees were done [19]. A fruit setting model can help us to optimize the heading practices for balancing the decrease in fruit numbers and the increase in mean fruit weight.

Our results also strongly recommend medium heading back as an appropriate pruning measure to reduce canopy crowding, to increase fruit size [29]. A possible mechanism for these effects is that medium heading can optimize resource allocation in relation to shoot and fruit growth, increase the availability of soluble carbohydrates to fruits.

Light heading favors fruit set in the peripheral zone of the canopy and significantly reduce light interception by the expanding shoots due to larger amounts of carbohydrates moving to the expanding shoots from the fruits. So, it is not a sustainable management measure to improve the profitability of nectarine, although it can increase the numbers of fruit on each parent branch.

Severe heading back is another heading practice, which removes most of flower buds that can set fruits and encourages the development of the current shoots. So, from a practical point of view, our results indicate that severe heading is to provide conditions favorable for vegetative growth of weak ageing trees in which a significant increase in flower bud initiation will reduce overall fruit set and inhibit the formation of next year's flower buds.

It is obviously that changes in the fruit setting pattern are caused by the heading practices, which presents additional evidence in support of architectural effects hypothesis.

Some pruning practices that work in one case might not work in another. Indeed, it is unclear whether different heading intensity could be declared to be appropriate when there is in fact limited information on its efficiency and effectiveness prior to heading. Experiments need to be run for virtual parent branches for which heading is completely harmless and simulation models, which in this case would be absolutely essential, output to determine whether they agree with the field observed data.
The major issues and their causes that are identified in an extremely intuitive way can help users understand the use of models and simulations in situations in which they do not have an opportunity to collect full information on heading practices.

\section{ACKNOWLEDGEMENTS}

This work was supported by the Natural Science Foundation of Guangdong Province (8151064001000009) and the Technology Research and Development Program of Guangdong Province, Contract No. 2016A020209003, 2008A020100026, 810302969091 , and 2009B091300161.

\section{REFERENCES}

[1] Arista M, Ortiz P L, and Talavera S. Apical pattern of fruit production in the racemes of Ceratonia siliqua (Leguminosae: Caesalpinioideae): role of pollinators. American Journal of Botany 1999; 86(12): 1708-1716. https://doi.org/10.2307/2656669

[2] Brunet J, Charlesworth D. Floral sex allocation in sequentially blooming plants. Evolution 1995; 49(1): 70-79. https://doi.org/10.1111/j.1558-5646.1995.tb05959.x

[3] Cieslak M, Cheddadi I, Boudon F, et al. Integrating Physiology and Architecture in Models of Fruit Expansion. Frontiers in Plant Science. 2016; 7: 1-19. https://doi.org/10.3389/fpls.2016.01739

[4] Cieslak M, Seleznyova AN, Hanan, J. A functional-structural kiwifruit vine model integrating architecture, carbon dynamics and effects of the environment. Annals of Botany 2011; 107(5): 747-764.

https://doi.org/10.1093/aob/mcq180

[5] Costes E, de Reffye P, Lichou J, Guedon Y, Audubert A, and Jay M. Stochastic modelling of apricot growth units and branching. Acta Horticulturae 1992; 313: 89-98. https://doi.org/10.17660/ActaHortic.1992.313.10

[6] Costes E, Guedon Y. Modeling the sylleptic branching on one-year-old trunks of apple cultivars. Journal of the American Society for Horticultural Science 1997; 122(1): 5362.

[7] Diggle PK, Architectural effects and the interpretation of patterns of fruit and seed development. Annual Review of Ecology and Systematics 1995; 26: 531-532.

https://doi.org/10.1146/annurev.es.26.110195.002531

[8] Fournier D, Costes E, Guedon Y, and Monet R. A comparison of different fruiting shoots of peach tree. Acta Horticulturae 1998; 465: 557-565. https://doi.org/10.17660/ActaHortic.1998.465.69

[9] Gloria DH, Dan M, Irene T, Li DH. Validation of PCREDAPOL: Fruit set prediction model for apples. Journal of Economic Entomology 1995; 88(4): 965-972. https://doi.org/10.1093/jee/88.4.965

[10] Godin C, Caraglio Y. A multiscale model of plant topological structures. Journal of Theoretical Biology 1998; 191(1): 1-46. https://doi.org/10.1006/jtbi.1997.0561

[11] Grossman YL, DeJong TM. Training and pruning system effects on vegetative growth potential light interception and cropping efficiency in peach trees. Journal of the American Society for Horticultural Science 1998; 123(6): 1058-1064.

[12] Guédon $Y$, Barthélémy $D$, Caraglio $Y$, Costes E. Pattern analysis in branching and axillary flowering sequences. Journal of Theoretical Biology. 2001; 212(4): 481-520. 
[13] Guitián J. Selective fruit abortion in Prunus mahaleb (Rosaceae). American Journal of Botany 1994; 81(12): 15551558. https://doi.org/10.1002/j.1537-2197.1994.tb11466.x

[14] Hall AJ, and Gandar PW. Stochastic models for fruit growth. Acta Horticulturae 1996; 416: 103-112. https://doi.org/10.17660/ActaHortic.1996.416.13

[15] Halle F, Oldeman RAA, and Tomlinson PB. Tropical trees and forests: an architectural analysis. Berlin. New York: Springer-Verlag.1978. https://doi.org/10.1007/978-3-642-81190-6

[16] Jaeger $\mathrm{M}$, and De Reffye PH. Basic concepts of computer simulation of plant growth. Journal of biosciences 1992; 19(3): 275-291. https://doi.org/10.1007/BF02703154

[17] Jourdan C, Rey H. Modelling and simulation of the architecture and development of the oil-palm (Elaeis guineensis Jacq.) root system. I. The model. Plant and Soil 1997; 190(2): 217-33.

https://doi.org/10.1023/A:1004218030608

[18] Kappel, F, Quamme, HA. Orchard training systems influence early canopy development and light microclimate within apple tree canopies. Canadian Journal of Plant Science 1993; 73(1): 237-248.

https://doi.org/10.4141/cjps93-038

[19] Lauri PÉ, Claverie J. Training sweet cherries to improve fruit size and quality-an overview of some recent concepts and practical aspects developed at INRA France. Acta Horticulturae 2005; 667: 361-366. https://doi.org/10.17660/ActaHortic.2005.667.51

[20] Lespinasse JM, Delortc F. Regulation of fruiting in apple. Role of the bourse and crowned brindles. Acta Horticulturae 1993; 349:229-246.

https://doi.org/10.17660/ActaHortic.1993.349.40

[21] Medrano M, Guitián P, and Guitián J. Patterns of fruit and seed set within inflorescences of Pancratium maritimum (Amaryllidaceae): nonuniform pollination resource limitation or architectural effects? American Journal of Botany 2000; 87(4): 493-501. https://doi.org/10.2307/2656592

[22] Menzel CM. The pattern and control of reproductive development in lychee: A review. Scientia Horticulturae 1984; 22(4): 333-345. https://doi.org/10.1016/S0304-4238(84)80005-9

[23] Myers SC. Structuring the young peach tree: Avoid wasteful pruning by following this training strategy. Fruit Grower 1994; 94(11): 10-13.
[24] Prusinkiewicz PW, Remphrey WR, Davidson CG, and HAMMEL MS. Modeling the architecture of expanding Fraxinus pennsylvanica stalks using L-systems. Canadian Journal of Botany 1994; 72(5): 701-714. https://doi.org/10.1139/b94-091

[25] Seleznyova AN, Thorp TG, Barnett AM, and Costes E. Quantitative analysis of shoot development and branching patterns in Actinidia. Annals of Botany 2002; 89(4): 471-482. https://doi.org/10.1093/aob/mcf069

[26] Smith GS, Curtis JP. A fast and effective method of measuring tree structure in 3 dimensions. Acta Horticulturae 1996; 416: 15-20. https://doi.org/10.17660/ActaHortic.1996.416.1

[27] Smith GS, Gravett IM, Edwards CM, Curtis JP, and Buwalda JG. Spatial Analysis of the Canopy of Kiwifruit Individual fruitbearing shoots as it Relates to the Physical Chemical and Postharvest Attributes of the Fruit. Annals of Botany 1994; (1): 99-111.

\section{https://doi.org/10.1006/anbo.1994.1012}

[28] Tustin S, Ferree D, Myers SL. et al. The international apple growth study. Acta Horticulturae 1997; 451: 693-699. https://doi.org/10.17660/ActaHortic.1997.451.83

[29] Willaume M, Lauri PE, and Sinoquet H. Light interception in apple trees influenced by canopy architecture manipulation. Trees: Structure and Function 2004; 18(6): 705-713. https://doi.org/10.1007/s00468-004-0357-4

[30] Wustenberg $\mathrm{H}$, and Keulemans J. A comparison of 3 plum tree training systems during the first five years of growth. Acta Horticulturae 1997; 625-631. https://doi.org/10.17660/ActaHortic.1997.451.73

[31] Xia N, Li BG, Guo Y, Deng XM. Modeling the branching patterns of peach tree branches (Prunus persica (L,) Batsch) after being pruned, Acta Botanica Sinica 2004; 46(7): 793802.

[32] Xia N, Li AS A process model for simulation the growth and development of sweet sorghum. Journal of Computer Science Technology Updates 2017; 4(2): 11-16 https://doi.org/10.15379/2410-2938.2017.04.02.01

[33] Xia N, Li AS, and Lin WJ. Simulation and Visualization of Nectarine Branching and Fruiting Responses to Pruning Using PrungingSim Software. Journal of Computer Science Technology Updates 2015; 2(1): 1-7. https://doi.org/10.15379/2410-2938.2015.02.01.01

\section{http://dx.doi.org/10.15379/2410-2938.2018.05.02.01}

(C) 2018 Xia and Li; Licensee Cosmos Scholars Publishing House.

This is an open access article licensed under the terms of the Creative Commons Attribution Non-Commercial License

(http://creativecommons.org/licenses/by-nc/3.0/), which permits unrestricted, non-commercial use, distribution and reproduction in any medium, provided the work is properly cited. 